\title{
BOUNDING SOLUTIONS OF PFAFF EQUATIONS
}

\author{
E. ESTEVES AND S. KLEIMAN \\ Instituto de Matemática Pura e Aplicada \\ Estrada Dona Castorina 110, \\ 22460-320 Rio de Janeiro RJ, Brazil \\ E-mail: esteves@impa.br \\ Department of Mathematics, MIT \\ 77 Massachusetts Avenue \\ Cambridge, MA 02139, USA \\ E-mail: kleiman@math.mit.edu
}

\begin{abstract}
Let $\omega$ be a Pfaff system of differential forms on $\mathbf{P}_{\mathbf{C}}^{n}$. Let $S$ be its singular locus, and $Y$ a solution of $\omega=0$. We prove $Y \cap S$ is of codimension at most 1 in $Y$, just as Jouanolou suspected; he proved this result assuming $\omega$ is completely integrable, and asked if the integrability is, in fact, needed. Furthermore, we prove a lower bound on the Castelnuovo-Mumford regularity of $Y \cap S$. As in two related articles, we derive upper bounds on numerical invariants of $Y$, thus contributing to the solution of the Poincaré problem. We work with Pfaff fields not necessarily induced by Pfaff systems, with ambient spaces more general than $\mathbf{P}_{\mathbf{C}}^{n}$, and usually in arbitrary characteristic.
\end{abstract}

\section{INTRODUCTION}

In his seminal work $[\mathrm{J}]$ on algebraic Pfaff equations, Jouanolou proved that a complex analytic foliation of positive dimension of an open subset $U \subseteq \mathbf{P}_{\mathbf{C}}^{n}$ has no compact leaves; see his Prop. 4.2, p.130. He went on, in his Cor. 4.2.7, p. 133, to prove that, if the foliation arises from a completely integrable system of Pfaff forms, if $U$ is the complement of the singular locus $S$ of the system, and if $Y \subseteq \mathbf{P}_{\mathrm{C}}^{n}$ is a closed subvariety such that $Y \cap U$ is a leaf, then $S$ intersects $Y$ in codimension at most 1. Then in Rem.4.2.8, p. 134, he said it would be interesting to remove the hypothesis of complete integrability.

The present article advances Jouanolou's work. As he envisioned, it is indeed possible to work with an arbitrary Pfaff system $\omega$ : without assuming

2000 Mathematics Subject Classification 37F75 (primary), 32S65, 14H99, 14B05, 14F10 (secondary).

Keywords Foliations, Pfaff equations, Poincaré problem, singularities, C-M regularity. 
integrability, we prove that the singular locus $S$ of $\omega$ intersects a solution $Y$ of $\omega=0$ in a subvariety of codimension at most 1 .

In fact, we go further. Let $X$ be, more generally, a complex projective scheme of pure dimension $n$. A Pfaff system on $X$ induces, via exterior powers and the perfect pairing of differential forms, a map $\eta: \Omega_{X}^{b} \rightarrow \mathcal{L}$ from the sheaf of differential forms to an invertible sheaf; see Subsec. 3.1. However, the converse is not true: such an $\eta$ does not come necessarily from a Pfaff system.

Let $\eta: \Omega_{X}^{b} \rightarrow \mathcal{L}$ be a nonzero map with $0<b<n$. Its singular locus is defined as the subscheme $S \subset X$ of points where $\eta$ is not surjective. Let $Y \subset X$ be a reduced closed subscheme of dimension $b$. Assume no $b$ dimensional component lies in $S$. Assume $Y$ is invariant under $\eta$; that is, $\eta \mid Y$ factors through the natural map $\Omega_{X}^{b} \mid Y \rightarrow \Omega_{Y}^{b}$. If $X$ is smooth, and $\eta$ arises from a Pfaff system, then $Y$ is a solution of the system in Jouanolou's sense; see Subsec. 3.1 and Prop. 3.2.

Under the above conditions, Prop. 3.3 says that the sheaf of ideals $\mathcal{J}$ of $Y \cap S$ in $X$ satisfies $\mathrm{H}^{b}(\mathcal{J} \otimes \mathcal{L}) \neq 0$ and that, if the induced map $\mathrm{H}^{b}(\eta): \mathrm{H}^{b}\left(\Omega_{X}^{b}\right) \rightarrow \mathrm{H}^{b}(\mathcal{L})$ vanishes, then $Y \cap S$ has codimension 1 in $Y$ and $\mathrm{h}^{b}(\mathcal{L} \mid Y)<\mathrm{h}^{b}\left(\Omega_{Y}^{b}\right)$.

If $X=\mathbf{P}_{\mathbf{C}}^{n}$, then $\mathrm{H}^{b}(\eta)=0$ because $\mathrm{H}^{b}(\mathcal{L})=0$; so $\operatorname{codim}(Y \cap S, Y)=1$. Furthermore, if $\mathrm{H}^{b}(\mathcal{J} \otimes \mathcal{L}) \neq 0$, then the Castelnuovo-Mumford regularity $\operatorname{reg}(Y \cap S)$ is greater than $m:=\operatorname{deg} \mathcal{L}+b$; see Cor. 4.5.

As Soares observed in the introduction to [S], Jouanolou's work can be used to tackle the Poincaré problem. Soares' observation served to motivate our work here and in [EK1] and [EK2].

In 1891, Poincaré [P], p. 161, posed the problem of bounding the degree of an algebraic curve $Y$ invariant under a polynomial vector field on the complex plane. Versions of this problem have been considered in a number of recent works; references are given in [EK1]. From our point of view, the general problem is simply to find upper bounds on the various numerical invariants of $Y$.

Roughly, Soares' idea is this: upper bounds on the numerical invariants of $Y$ arise from lower bounds on the numerical invariants of $Y \cap S$, where, as always, $S$ is the singular locus of the field. In [EK1], this idea is used to improve bounds obtained by Campillo, Carnicer, and García de la Fuente [CCG], and by Du Plessis and Wall [dPW].

In the present article, we approach the Poincaré problem in a new way. It is based on the inequality $\mathrm{h}^{b}(\mathcal{L} \mid Y)<\mathrm{h}^{b}\left(\Omega_{Y}^{b}\right)$, which obtains if $\mathrm{H}^{b}(\eta)=0$ according to Prop. 3.3. Our Cor. 4.5 gives one application: if $X=\mathbf{P}_{\mathbf{C}}^{n}$, if $Y$ is arithmetically Cohen-Macaulay - for instance, a complete intersection and if $\mathrm{h}^{b}\left(\Omega_{Y}^{b}\right)=1$, then $\operatorname{reg}(Y) \leq m+1$. The third condition $\mathrm{h}^{b}\left(Y, \Omega_{Y}^{b}\right)=1$ is satisfied when $Y$ is integral and has normal-crossings in codimension 1; see Rem. 4.7. Since the regularity of a plane curve is just its degree, we recover a 
fundamental result proved by Cerveau and Lins Neto [CL]. We also recover [E], Thm. 1, p. 3, which generalizes their result to curves in $\mathbf{P}_{\mathbf{C}}^{n}$.

The condition $\mathrm{h}^{b}\left(Y, \Omega_{Y}^{b}\right)=1$ is also satisfied when $Y$ has higher singularities, yielding new solutions to the original Poincaré problem on $\mathbf{P}_{\mathbf{C}}^{2}$. Indeed, assume $Y$ is a plane curve of degree $d$. Let $\Sigma$ be its singular locus, the subscheme cut out by its polars. Set $\sigma:=\operatorname{reg}(\Sigma)$. In [EK2], Thm. 2.5 asserts that $d \leq m+1$ if $\sigma \leq d-2$; otherwise, $2 d \leq m+\sigma+3$, with equality if $d \geq 2 m$ and $S$ is finite.

Our Prop. 3.3 applies to ambient varieties other than projective space. For instance, it applies to multiprojective space; see Thm. 4.3.

Proceeding in a different direction, assume $X$ is smooth and $\operatorname{Pic}(X)=\mathbf{Z}$. We obtain two results. First, Prop. 3.4 says that, if the normal sheaf of $Y$ in $X$ has positive degree on some curve lying in the smooth locus of $Y$, then $\operatorname{codim}(Y \cap S, Y)=1$. Second, Thm. 3.6 says that, if $Y$ is a hypersurface with normal-crossings in codimension 1 , then $\operatorname{deg} Y \leq \operatorname{deg} \mathcal{L}(-K)$ where $K$ is a canonical divisor of $X$. This theorem generalizes part of the main theorem in $[\mathrm{BM}]$, p. 594 .

Using methods similar to Jouanolou's, Lehmann [Le] too advanced his work. However, our results seem to be completely independent of Lehmann's; and our methods, completely different.

Surprisingly, our results rest on a rather unsurprising fact: the map $\mathrm{H}^{b}\left(\Omega_{X}^{b}\right) \rightarrow \mathrm{H}^{b}\left(\Omega_{Y}^{b}\right)$ does not vanish. This nonvanishing was known in some generality, at a minimum when $X$ and $Y$ are smooth; and probably it was expected in the generality we need. However, there appears to be no suitable reference. Some references are too abstract; others, not general enough. So the fact is proved in Prop. 2.1.

All our schemes are defined over a fixed algebraically closed field. All our results hold over any field of characteristic 0, not just $\mathbf{C}$. Except for Prop. 3.4 and Thm. 3.6, all our results hold over a field of characteristic $p>0$ if the restriction map $\mathrm{H}^{b}\left(\Omega_{X}^{b}\right) \rightarrow \mathrm{H}^{b}\left(\Omega_{Y}^{b}\right)$ does not vanish. Prop. 2.2 gives sufficient conditions for this nonvanishing. For instance, if $X=\mathbf{P}^{n}$, then it is enough that $p \nmid \operatorname{deg} Y$. The proof of Prop. 2.2 is similar to that of Prop. 2.1, but is more involved, most notably in its use of the theory of residues. We feel the effort is worthwhile, owing to the resurgence of interest in foliations in positive characteristic, caused by McQuillan's proof in $[\mathrm{M}]$ of the Green-Griffiths conjecture, which uses Miyaoka's results proved by means of reduction to positive characteristic.

\section{NONVANISHING}

Proposition 2.1. Let $X$ be a projective scheme over a field of characteristic zero, and $f: Y \rightarrow X$ a finite map. Set $b:=\operatorname{dim} Y$. Then the natural map $\mathrm{H}^{b}\left(\Omega_{X}^{b}\right) \rightarrow \mathrm{H}^{b}\left(\Omega_{Y}^{b}\right)$ is nonzero.

Proof. We proceed by induction on $b$. If $b=0$, then the map in question 
is just the pullback map $\mathrm{H}^{0}\left(\mathcal{O}_{X}\right) \rightarrow \mathrm{H}^{0}\left(\mathcal{O}_{Y}\right)$, which is always nonzero. So assume $b>0$.

Let $Y^{\prime} \subseteq Y$ be an irreducible component of dimension $b$, and give $Y^{\prime}$ the reduced structure. It is enough to show the composition

$$
\mathrm{H}^{b}\left(\Omega_{X}^{b}\right) \rightarrow \mathrm{H}^{b}\left(\Omega_{Y}^{b}\right) \rightarrow \mathrm{H}^{b}\left(\Omega_{Y^{\prime}}^{b}\right)
$$

is nonzero. So we may replace $Y$ by $Y^{\prime}$, and thus assume $Y$ is integral.

Let $\pi: Y^{*} \rightarrow Y$ be the normalization map. It is enough to show the natural map $\mathrm{H}^{b}\left(\Omega_{X}^{b}\right) \rightarrow \mathrm{H}^{b}\left(\Omega_{Y^{*}}^{b}\right)$ is nonzero. Since $\pi$ is finite and, hence, $\operatorname{dim} Y^{*}=b$, we may replace $Y$ by $Y^{*}$, and thus assume $Y$ is normal.

Let us now find on $X$ an effective Cartier divisor $E$ satisfying the following conditions:

(2.1.1) The preimage $F:=f^{-1}(E)$ is nonempty, Cartier and smooth in codimension 1.

(2.1.2) No component of the singular locus of $Y$ is contained in $F$.

(2.1.3) The induced maps $\Omega_{X}^{b} \rightarrow \Omega_{X}^{b}(E)$ and $\Omega_{Y}^{b} \rightarrow \Omega_{Y}^{b}(F)$ are injective.

(2.1.4) If $b>1$, then $\mathrm{H}^{b-1}\left(\Omega_{Y}^{b}(F)\right)=0$.

To start, let $E$ be any effective very ample divisor such that $E \not \supset f(Y)$. However, if $b>1$, then take $E$ ample enough so that $\mathrm{H}^{b-1}\left(f_{*} \Omega_{Y}^{b}(E)\right)=0$. Then $F:=f^{-1}(E)$ is Cartier on $Y$. Hence (2.1.4) holds. Moreover, $F$ is nonempty because $E$ is ample and $\operatorname{dim} f(Y)=b>0$.

Vary $E$ inside its complete linear system, keeping $E \not \supset f(Y)$. Correspondingly, $F$ traces on $Y$ a linear system without base points (although it may be incomplete). If $E$ is general, then $E$ and $F$ contain no associated point of $\Omega_{X}^{b}$ and $\Omega_{Y}^{b}$ respectively; hence, (2.1.3) holds. Similarly, (2.1.2) holds if $E$ is general.

Finally, since the characteristic is 0 , if $E$ is general, then $F$ is smooth off the singular locus of $Y$ by a form of Bertini's first theorem; see [K], Cor. 5, p. 291. In particular, $F$ is smooth in codimension 1 by (2.1.2). Then (2.1.1) holds.

Consider now the second fundamental exact sequence:

$$
\mathcal{O}_{X}(-E)\left|E \rightarrow \Omega_{X}^{1}\right| E \rightarrow \Omega_{E}^{1} \rightarrow 0 .
$$

In a standard way, it induces a map,

$$
\eta_{E, X}: \Omega_{E}^{b-1} \rightarrow \Omega_{X}^{b}(E) \mid E ;
$$

namely, given the germ of a form on $E$, lift it to $X$, then wedge with the meromorphic 1 -form $d t / t$ where $t=0$ is a local equation for $E$, and finally restrict to $E$.

Tensoring the standard exact sequence

$$
0 \rightarrow \mathcal{O}_{X} \rightarrow \mathcal{O}_{X}(E) \rightarrow \mathcal{O}_{X}(E) \mid E \rightarrow 0
$$

with $\Omega_{X}^{b}$, we obtain a sequence

$$
0 \rightarrow \Omega_{X}^{b} \rightarrow \Omega_{X}^{b}(E) \rightarrow \Omega_{X}^{b}(E) \mid E \rightarrow 0
$$


which is exact on the left by (2.1.3) above. Form the coboundary map

$$
h_{E, X}: \mathrm{H}^{b-1}\left(\Omega_{X}^{b}(E) \mid E\right) \rightarrow \mathrm{H}^{b}\left(\Omega_{X}^{b}\right),
$$

and set $v_{E, X}:=h_{E, X} \circ \mathrm{H}^{b-1}\left(\eta_{E, X}\right)$.

Similarly, for $Y$ and $F$, we have maps $\eta_{F, Y}, h_{F, Y}$ and $v_{F, Y}$. Form the diagram

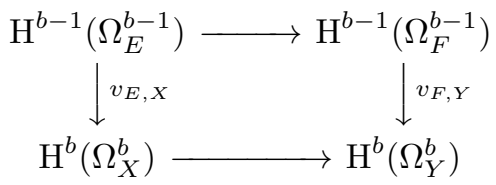

using the natural horizontal maps. It is plainly commutative.

By induction, the top map is nonzero. Now, $F$ is smooth in codimension 1 by (2.1.1). In addition, the singular locus of $Y$ intersects $F$ in codimension 2 by (2.1.2). Therefore, $\eta_{F, Y}: \Omega_{F}^{b-1} \rightarrow \Omega_{Y}^{b}(F) \mid F$ is an isomorphism in codimension 1. Hence $\mathrm{H}^{b-1}\left(\eta_{F, Y}\right)$ is an isomorphism.

First assume $b>1$. Then $\mathrm{H}^{b-1}\left(\Omega_{Y}^{b}(F)\right)=0$ by (2.1.4). So the coboundary map

$$
h_{F, Y}: \mathrm{H}^{b-1}\left(\Omega_{Y}^{b}(F) \mid F\right) \rightarrow \mathrm{H}^{b}\left(\Omega_{Y}^{b}\right)
$$

is injective. Hence, in Diagram (2.1.5), the top-right composition is nonzero. Hence the left-bottom composition is also. Therefore, the bottom map is nonzero.

Finally, assume $b=1$. In this case, Diagram (2.1.5) becomes

$$
\begin{aligned}
\mathrm{H}^{0}\left(\mathcal{O}_{E}\right) & \longrightarrow \mathrm{H}^{0}\left(\mathcal{O}_{F}\right) \\
\downarrow v_{E, X} & \downarrow v_{F, Y} \\
\mathrm{H}^{1}\left(\Omega_{X}^{1}\right) & \longrightarrow \mathrm{H}^{1}\left(\Omega_{Y}^{1}\right)
\end{aligned}
$$

As before, we need only show that the top-right composition is nonzero. To do so, we need only prove $v_{F, Y}(1) \neq 0$.

By definition, $v_{F, Y}$ is the following composition:

$$
\mathrm{H}^{0}\left(\mathcal{O}_{F}\right) \stackrel{\mathrm{H}^{0}\left(\eta_{F, Y}\right)}{\longrightarrow} \mathrm{H}^{0}\left(\Omega_{Y}^{1}(F) \mid F\right) \stackrel{h_{F, Y}}{\longrightarrow} \mathrm{H}^{1}\left(\Omega_{Y}^{1}\right) .
$$

Given $y \in F$, let $t$ be a uniformizing parameter of $F$ at $y$. Then $\eta_{F, Y}(1)$ is at $y$ equal to the class of $d t / t$. Now, let $\rho_{Y}: \mathrm{H}^{1}\left(\Omega_{Y}^{1}\right) \rightarrow k$ be the global residue map; we compute it by summing local residues. Consequently, $\rho_{Y}\left(v_{F, Y}(1)\right)=\operatorname{deg} F$. Since $F$ is nonempty, $\operatorname{deg} F \neq 0$. Hence $v_{F, Y}(1) \neq 0$, and the proof is complete.

Proposition 2.2. Let $X$ be a projective scheme over a field of characteristic $p>0$, and $f: Y \rightarrow X$ a finite map. Set $b:=\operatorname{dim} Y$. Assume there are Cartier divisors $E_{1}, \ldots, E_{b}$ on $X$ such that

$$
\int_{X} E_{1} \cdots E_{b} \cdot f_{*}[Y] \not \equiv 0(\bmod p) .
$$


Then the natural map $\mathrm{H}^{b}\left(\Omega_{X}^{b}\right) \rightarrow \mathrm{H}^{b}\left(\Omega_{Y}^{b}\right)$ is nonzero.

Proof. The proof is analogous to that of Proposition 2.1 (and reproves the proposition); we dwell only on the alterations. They are required because we can no longer guarantee $F$ is smooth in codimension 1. Notably, we must use more of the theory of residues.

As before, we may assume that $b>0$. Again, we may replace $Y$ by some integral component $Y^{\prime}$; indeed, (2.2.1) will still hold as $[Y]$ is a linear combination of the $\left[Y^{\prime}\right]$ of dimension $b$. Then $f$ is generically étale; indeed, if $n:=\operatorname{deg} f$, then $f_{*}[Y]=n[f(Y)]$, and so $p \nmid n$ owing to (2.2.1).

So we may assume that $Y$ is generically smooth of pure dimension $b$ and that $f$ is generically étale onto its image. We are going to prove a stronger assertion, namely the nonvanishing of the composition

$$
\mathrm{H}^{b}\left(\Omega_{X}^{b}\right) \longrightarrow \mathrm{H}^{b}\left(\Omega_{Y}^{b}\right) \stackrel{\rho_{Y}}{\longrightarrow} k .
$$

Here $\rho_{Y}$ is the generalized residue map, defined as explained in the next paragraph.

Given an integral, projective scheme $Z$ of dimension $e$, let us denote by $\rho_{Z}: \mathrm{H}^{e}\left(\Omega_{Z}^{e}\right) \rightarrow k$ its generalized residue map; see Thm. 0.1 on p. 10 of [Li] and the discussion thereafter, where $\rho_{Z}$ is denoted by $\int_{Z}$ however. Given a generically smooth, projective scheme $Z$ of pure dimension $e$, let $\rho_{Z}$ denote the composition

$$
\mathrm{H}^{e}\left(\Omega_{Z}^{e}\right) \rightarrow \mathrm{H}^{e}\left(\Omega_{Z_{1}}^{e}\right) \oplus \cdots \oplus \mathrm{H}^{e}\left(\Omega_{Z_{s}}^{e}\right) \stackrel{\left(\rho_{Z_{1}}, \ldots, \rho_{Z_{s}}\right)}{\longrightarrow} k,
$$

where $Z_{1}, \ldots, Z_{s}$ are the irreducible components of $Z$ with their reduced structures, and the first map is the natural one.

As in the proof of Proposition 2.1, we can find an effective very ample divisor $E$ on $X$ such that $F:=f^{-1}(E)$ is Cartier, nonempty, and (2.1.2) and (2.1.3) hold. In addition, as we are going to see, we may assume the following three conditions hold:

(2.2.3) We have $\int E \cdot E_{2} \cdots E_{b} \cdot f_{*}[Y] \not \equiv 0(\bmod p)$.

(2.2.4) The scheme $F$ is generically smooth and $f \mid F$ is generically étale onto its image.

(2.2.5) There are a finite map $g: Y \rightarrow P$, where $P:=\mathbf{P}^{b}$, and a hyperplane $M \subset P$ such that $g^{-1} M=F$ and $g \mid F$ is generically étale onto $M$.

If (2.2.3) doesn't already hold, then replace $E$ by a general member of the linear system $\left|m E+E_{1}\right|$ for $m \gg 0$. Then (2.1.2), (2.1.3) and (2.2.3) hold.

As to (2.2.4), since generically $f$ is étale and $Y$ is smooth, $Y$ has a smooth, dense open subset $U$ such that $f \mid U$ is étale over $f(Y)$. We may replace $E$ by a general member of the linear system $|E|$, and assume that every component of $F$ intersects $U$. Furthermore, even though $p>0$, we may assume $F \cap U$ is smooth by another form of Bertini's first theorem; see [K], Cor. 12, p. 296. Then $F$ is generically smooth and $f \mid F$ is generically étale onto $Z:=f(F)$. 
To ensure (2.2.5), use the system $|E|$ to embed $X$ in a projective space $P^{\prime}$, and let $E^{\prime}$ be a hyperplane that cuts $E$ out of $X$. Let $z_{1}, \ldots, z_{s} \in Z$ be simple points, one for each component of $Z$. Let $C^{\prime}$ be a linear subspace of $E^{\prime}$ of codimension $d$ such that $C^{\prime}$ misses both $Z$ and its tangent spaces $T_{z_{i}} Z \subset E^{\prime}$. Then $C^{\prime}$ misses $f(Y)$ too. So projection from $C^{\prime}$ induces a finite map $g^{\prime}: f(Y) \rightarrow \mathbf{P}^{b}$. Set $P:=\mathbf{P}^{b}$ and $g:=g^{\prime} f$.

There is a hyperplane $M \subset P$ such that $g^{\prime-1} M=Z$ since $C^{\prime} \subset E^{\prime}$. Hence $g^{\prime} \mid Z$ is finite onto $M$. It is also étale at each $z_{i}$ since $C^{\prime}$ misses $T_{z_{i}} Z$. In particular, $g^{\prime} \mid Z$ is generically étale onto $M$. Since also $f \mid F$ is generically étale onto its image, by (2.2.4), the composition $g \mid F$ is generically étale onto $M$. Thus (2.2.5) holds.

We proceed by induction on $b \geq 1$, using the diagrams of maps (2.1.5) and (2.1.6), which exist and are commutative by (2.1.3).

First assume $b=1$. Since Diagram (2.1.6) is commutative, we need only prove that $\rho_{Y}\left(v_{F, Y}(1)\right) \neq 0$. Now, $Y$ is smooth along $F$ by (2.1.2). Given $y \in F$, let $t$ be a uniformizing parameter of $F$ at $y$. Since $F$ is of pure dimension 0 and generically smooth by (2.2.4), $F$ is reduced. Then, as before, $\eta_{F, Y}(1)$ is at $y$ equal to the class of $d t / t$. Consequently, $\rho_{Y}\left(v_{F, Y}(1)\right)=\operatorname{deg} F$ in $k$. However, $\operatorname{deg} F=\int E \cdot f_{*}[Y]$. Hence (2.2.3) implies $\rho_{Y}\left(v_{F, Y}(1)\right) \neq 0$, as desired.

Finally, assume $b>1$. Since $F$ is generically smooth with pure dimension $b-1$, and $f \mid F$ is generically étale onto its image, by induction the composition

$$
\mathrm{H}^{b-1}\left(\Omega_{E}^{b-1}\right) \longrightarrow \mathrm{H}^{b-1}\left(\Omega_{F}^{b-1}\right) \stackrel{\rho_{F}}{\longrightarrow} k
$$

is nonzero. Now, Diagram (2.1.5) is commutative. It will follow that the composition (2.2.2) is nonzero once we prove that the following diagram is commutative:

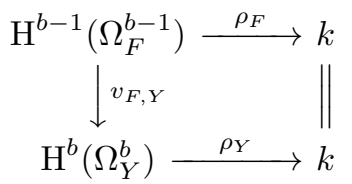

We are going to reduce the matter to the case where $M$ and $P$ replace $F$ and $Y$.

Using the natural maps, form the following diagram:

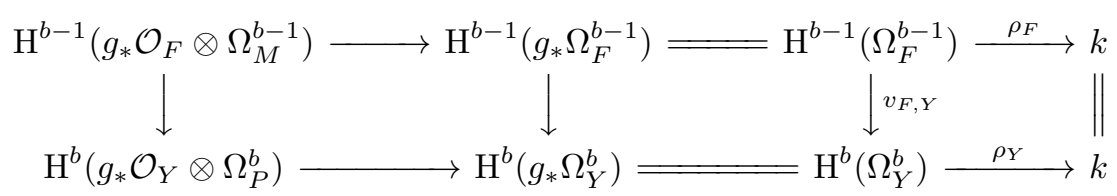

A look at the construction of the left-hand square shows it is commutative. Its top map is surjective; indeed, $g \mid F$ is generically étale onto $M$ by (2.2.5), so $g_{*} \mathcal{O}_{F} \otimes \Omega_{M}^{b-1} \rightarrow g_{*} \Omega_{F}^{b-1}$ is generically surjective. It will follow that the 
right-hand square is commutative once we prove that the outer "square" is commutative.

Let $Y_{1}, \ldots, Y_{t}$ be the irreducible components of $Y$ with their reduced structure. The bottom composition in (2.2.6) is equal to the following composition of natural maps:

$$
\mathrm{H}^{b}\left(g_{*} \mathcal{O}_{Y} \otimes \Omega_{P}^{b}\right) \rightarrow \bigoplus_{i=1}^{t} \mathrm{H}^{b}\left(g_{*} \mathcal{O}_{Y_{i}} \otimes \Omega_{P}^{b}\right) \rightarrow \bigoplus_{i=1}^{t} \mathrm{H}^{b}\left(\Omega_{Y_{i}}^{b}\right) \stackrel{\left(\rho_{Y_{1}}, \ldots, \rho_{Y_{t}}\right)}{\longrightarrow} k .
$$

By Thm. 0.1(b) on p. 10 of [Li], for each $i$ the diagram below commutes:

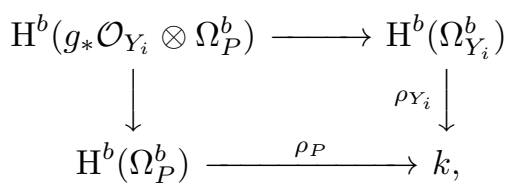

where the left vertical map is induced by the trace map $g_{*} \mathcal{O}_{Y_{i}} \rightarrow \mathcal{O}_{P}$. Since $Y$ is generically smooth, the trace map $g_{*} \mathcal{O}_{Y} \rightarrow \mathcal{O}_{P}$ is the sum of the trace maps $g_{*} \mathcal{O}_{Y_{i}} \rightarrow \mathcal{O}_{P}$. So the bottom composition in (2.2.6) is equal to the bottom composition below:

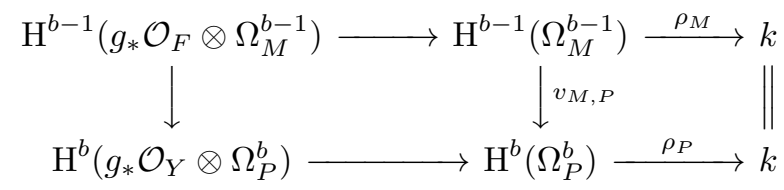

where the left-hand horizontal maps are induced by the two trace maps $g_{*} \mathcal{O}_{F} \rightarrow \mathcal{O}_{M}$ and $g_{*} \mathcal{O}_{Y} \rightarrow \mathcal{O}_{P}$. The latter map restricts to the former, and it follows that the left-hand square is commutative. By analogy, the top composition in (2.2.6) is equal to that in (2.2.7). So the outer "square" in (2.2.6) is commutative if the right-hand square in (2.2.7) is commutative.

By Thm. 0.1(a) on p. 10 of [Li], $\rho_{M}$ and $\rho_{P}$ are the "well-known canonical isomorphisms." A simple explicit calculation now shows the right-hand square in (2.2.7) is commutative.

\section{PFAFF FIELDS}

3.1. Pfaff systems, equations, and fields. A Pfaff system of rank a on a smooth scheme $X$ of pure dimension $n$ over a field is, according to Jouanolou $[\mathrm{J}]$, pp. 136-38, a nonzero map $u: \mathcal{E} \rightarrow \Omega_{X}^{1}$ where $\mathcal{E}$ is a locally free sheaf of constant rank $a$ with $0<a<n$. The singular locus of the system $u$ is the closed subscheme $S$ of $X$ whose ideal $\mathcal{I}_{S}$ is the image of the induced map $\bigwedge^{a} \mathcal{E} \otimes\left(\Omega_{X}^{a}\right)^{*} \rightarrow \mathcal{O}_{X}$. A solution is a closed subscheme $Y$ of $X$ with pure codimension $a$ such that the map

$$
\left(\bigwedge^{a} d\right) \wedge\left(\bigwedge^{a} u \mid Y\right): \bigwedge^{a}\left(\mathcal{I}_{Y, X} / \mathcal{I}_{Y, X}^{2}\right) \otimes \bigwedge^{a} \mathcal{E}\left|Y \rightarrow \bigwedge^{2} \Omega_{X}^{a}\right| Y
$$


vanishes, where $d: \mathcal{I}_{Y, X} / \mathcal{I}_{Y, X}^{2} \rightarrow \Omega_{X}^{1} \mid Y$ is the standard map, given by differentiation.

The notions of singular locus and solution involve the map $\bigwedge^{a} u$, not $u$ directly. So it is natural to generalize the theory in the following way; compare with Brunella and Mendes $[\mathrm{BM}]$, pp. 593-94. Define a Pfaff equation of rank $a$ to be an equation $\sigma=0$ where $\sigma$ is a nonzero global section of $\Omega_{X}^{a} \otimes \mathcal{N}$ for a given integer $a$ and a given invertible sheaf $\mathcal{N}$. The singular locus is the closed subscheme whose ideal is the image of the dual map $\left(\Omega_{X}^{a}\right)^{*} \otimes \mathcal{N}^{*} \rightarrow \mathcal{O}_{X}$. A solution is a closed subscheme $Y$ with pure codimension $a$ such that the following natural map vanishes:

$$
\left(\bigwedge^{a} d\right) \wedge\left(\sigma \otimes \mathcal{N}^{*}\right)\left|Y: \bigwedge^{a}\left(\mathcal{I}_{Y, X} / \mathcal{I}_{Y, X}^{2}\right) \otimes \mathcal{N}^{*}\right| Y \rightarrow \bigwedge^{2} \Omega_{X}^{a} \mid Y .
$$

Alternatively, we may view a Pfaff equation as follows. Set $b:=n-a$. Let $\tau: \Omega_{X}^{a} \otimes \Omega_{X}^{b} \rightarrow \Omega_{X}^{n}$ be the natural pairing; $\tau$ is perfect since $X$ is smooth of pure dimension $n$. Set $\mathcal{L}:=\Omega_{X}^{n} \otimes \mathcal{N}$. Then there is a natural isomorphism,

$$
\mathrm{H}^{0}\left(\Omega_{X}^{a} \otimes \mathcal{N}\right)=\operatorname{Hom}\left(\Omega_{X}^{b}, \mathcal{L}\right),
$$

under which $\sigma$ corresponds to the composition $\eta:=(\tau \otimes \mathcal{N}) \circ\left(\sigma \otimes \Omega_{X}^{b}\right)$. Thus giving $(a, \mathcal{N}, \sigma)$ is equivalent to giving $(b, \mathcal{L}, \eta)$.

More generally, without assuming that $X$ is smooth or equidimensional, define a Pfaff field of rank $b$ to be a nonzero map $\eta: \Omega_{X}^{b} \rightarrow \mathcal{L}$ for a given integer $b$ with $0<b<n$ and a given invertible sheaf $\mathcal{L}$. Define the singular locus $S$ of $\eta$ to be the closed subscheme of $X$ whose ideal $\mathcal{I}_{S}$ is the image of the induced map $\Omega_{X}^{b} \otimes \mathcal{L}^{*} \rightarrow \mathcal{O}_{X}$. Say that a closed subscheme $Y$ of $X$ is invariant under $\eta$ if the restriction $\eta\left|Y: \Omega_{X}^{b}\right| Y \rightarrow \mathcal{L} \mid Y$ factors through the standard map $\beta: \Omega_{X}^{b} \mid Y \rightarrow \Omega_{Y}^{b}$, in other words, if there is a commutative diagram

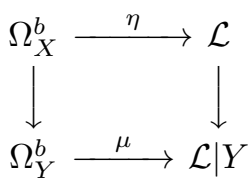

whose vertical maps are the standard maps.

Again assume that $X$ is smooth of pure dimension $n$. Then it is easy to see that the singular locus of the Pfaff field $\eta$ is the same as the singular locus of the corresponding Pfaff equation $\sigma=0$. Now, to avoid uninteresting cases, assume $Y$ is reduced and equidimensional and no component lies in $S$. Then $Y$ is invariant under $\eta$ if and only if $Y$ is a solution of $\sigma=0$, at least when $\operatorname{dim} Y=b$, according to the next proposition.

Proposition 3.2. Let $X$ be a smooth equidimensional scheme, $\eta: \Omega_{X}^{b} \rightarrow \mathcal{L}$ a Pfaff field, $S$ its singular locus, and $\sigma=0$ the corresponding Pfaff equation. Let $Y$ be a closed subscheme; assume $Y$ is reduced of pure dimension $b$ and no component lies in $S$. Then $Y$ is invariant under $\eta$ if and only if $Y$ is a solution of $\sigma=0$. 
Proof. Since no component of $Y$ lies in $S$, there is an open subset $U$ of $X-S$ such that $V:=U \cap Y$ is dense in $Y$. Since $Y$ is reduced, we may assume $V$ is smooth. Moreover, $Y$ is invariant under $\eta$ if and only if $V$ is invariant under $\eta \mid U$; indeed, if we let $\mathcal{K}$ denote the kernel of the map $\Omega_{X}^{b} \mid Y \rightarrow \Omega_{Y}^{b}$, then $(\eta \mid Y)(\mathcal{K})$ vanishes if and only if its restriction over $V$ vanishes, since $\mathcal{L}$ is invertible and $Y$ is reduced. Similarly, $Y$ is a solution of $\sigma=0$ if and only if $V$ is a solution of $\sigma \mid U=0$; indeed, the image of the map in (3.1.1) vanishes if and only if its restriction over $V$ vanishes. Replacing $X$ by $U$ and $Y$ by $V$, we may thus assume $Y$ is smooth and $S$ is empty.

Let $n:=\operatorname{dim} X$ and $a:=n-b$. Set $\mathcal{N}:=\mathcal{L} \otimes\left(\Omega_{X}^{n}\right)^{-1}$ and $\mathcal{J}:=\mathcal{I}_{Y, X}$. Since $X$ and $Y$ are smooth, $d: \mathcal{J} / \mathcal{J}^{2} \rightarrow \Omega_{X}^{1} \mid Y$ is locally split injective; whence, so is $\bigwedge^{a} d$. Since $S$ is empty, $\sigma \otimes \mathcal{N}^{*}: \mathcal{N}^{*} \rightarrow \Omega_{X}^{a}$ is locally split injective too; whence, so is its restriction to $Y$. It follows that the map in (3.1.1) vanishes if and only if there is a map, necessarily an isomorphism, $\zeta: \mathcal{N}^{*} \mid Y \rightarrow \bigwedge^{a}\left(\mathcal{J} / \mathcal{J}^{2}\right)$ such that $\bigwedge^{a} d \circ \zeta$ is equal to $\left(\sigma \otimes \mathcal{N}^{*}\right) \mid Y$. In other words, $Y$ is a solution of $\sigma=0$ if and only if such a $\zeta$ exists.

On the other hand, consider the map $\beta: \Omega_{X}^{b} \mid Y \rightarrow \Omega_{Y}^{b}$, and form the composition

$$
\alpha: \mathcal{N}^{*} \otimes \Omega_{X}^{b} \stackrel{\left(\sigma \otimes \mathcal{N}^{*}\right) \otimes \Omega_{X}^{b}}{\longrightarrow} \Omega_{X}^{a} \otimes \Omega_{X}^{b} \stackrel{\tau}{\longrightarrow} \Omega_{X}^{n}
$$

where $\tau$ is the natural pairing of forms. Virtually by definition, $Y$ is invariant under $\eta$ if and only if there is a map $\gamma: \mathcal{N}^{*}\left|Y \otimes \Omega_{Y}^{b} \rightarrow \Omega_{X}^{n}\right| Y$ such that $\alpha \mid Y=\gamma \circ\left(\mathcal{N}^{*} \mid Y \otimes \beta\right)$.

Since $X$ and $Y$ are smooth, the natural map $\psi: \bigwedge^{a}\left(\mathcal{J} / \mathcal{J}^{2}\right) \otimes \Omega_{Y}^{b} \rightarrow \Omega_{X}^{n} \mid Y$ is an isomorphism such that

$$
\tau \mid Y \circ\left(\bigwedge^{a} d \otimes \Omega_{X}^{b} \mid Y\right)=\psi \circ\left(\bigwedge^{a}\left(\mathcal{J} / \mathcal{J}^{2}\right) \otimes \beta\right) .
$$

Since $\Omega_{Y}^{b}$ is invertible, every map $\gamma: \mathcal{N}^{*}\left|Y \otimes \Omega_{Y}^{b} \rightarrow \Omega_{X}^{n}\right| Y$ can be written in the form $\gamma=\psi \circ\left(\zeta \otimes \Omega_{Y}^{b}\right)$ where $\zeta: \mathcal{N}^{*} \mid Y \rightarrow \bigwedge^{a}\left(\mathcal{J} / \mathcal{J}^{2}\right)$. Hence $Y$ is invariant under $\eta$ if and only if there exists a $\zeta$ such that $\alpha \mid Y=\psi \circ\left(\zeta \otimes \Omega_{Y}^{b}\right) \circ\left(\mathcal{N}^{*} \mid Y \otimes \beta\right)$.

Given $\zeta$, using the funtoriality of $\otimes$ twice and Equation (3.2.1), we obtain

$$
\begin{aligned}
\psi \circ\left(\zeta \otimes \Omega_{Y}^{b}\right) \circ\left(\mathcal{N}^{*} \mid Y \otimes \beta\right) & =\psi \circ\left(\bigwedge^{a}\left(\mathcal{J} / \mathcal{J}^{2}\right) \otimes \beta\right) \circ\left(\zeta \otimes \Omega_{X}^{b} \mid Y\right) \\
& =\tau \mid Y \circ\left(\bigwedge^{a} d \otimes \Omega_{X}^{b} \mid Y\right) \circ\left(\zeta \otimes \Omega_{X}^{b} \mid Y\right) \\
& =\tau \mid Y \circ\left(\left(\bigwedge^{a} d \circ \zeta\right) \otimes \Omega_{X}^{b} \mid Y\right) .
\end{aligned}
$$

Now, $\alpha=\tau \circ\left(\left(\sigma \otimes \mathcal{N}^{*}\right) \otimes \Omega_{X}^{b}\right)$ by definition, and $\tau \mid Y$ is a perfect pairing because $X$ is smooth. Hence, by the preceding paragraph, $Y$ is invariant under $\eta$ if and only if there exists a $\zeta$ such that $\bigwedge^{a} d \circ \zeta$ is equal to $\left(\sigma \otimes \mathcal{N}^{*}\right) \mid Y$. By the second paragraph, such a $\zeta$ exists if and only if $Y$ is a solution of $\sigma=0$.

Proposition 3.3. Let $X$ be a projective scheme, $Y \subseteq X$ a reduced closed subscheme of dimension b such that the induced map $\overline{\mathrm{H}}^{b}\left(\Omega_{X}^{b}\right) \rightarrow \mathrm{H}^{b}\left(\Omega_{Y}^{b}\right)$ is 
nonzero. Let $\eta: \Omega_{X}^{b} \rightarrow \mathcal{L}$ be a Pfaff field, $S$ its singular locus. Assume that no b-dimensional component of $Y$ lies in $S$ and that $Y$ is invariant under $\eta$. Then $\mathrm{H}^{b}\left(\mathcal{I}_{Y \cap S, X} \otimes \mathcal{L}\right) \neq 0$.

Furthermore, if $\mathrm{H}^{b}(\eta)=0$, then the following three statements hold:

(1) The restriction map $\mathrm{H}^{b-1}(\mathcal{L}) \rightarrow \mathrm{H}^{b-1}(\mathcal{L} \mid(Y \cap S))$ is not surjective.

(2) The intersection $Y \cap S$ has maximal dimension, $\operatorname{dim}(Y \cap S)=b-1$.

(3) The induced map $\mathrm{H}^{b}\left(\Omega_{Y}^{b}\right) \rightarrow \mathrm{H}^{b}(\mathcal{L} \mid Y)$ is surjective, but not bijective.

Proof. Let $\mu: \Omega_{Y}^{b} \rightarrow \mathcal{L} \mid Y$ be the map making Diagram (3.1.2) commute. First, let us see that Diagram (3.1.2) induces a commutative diagram

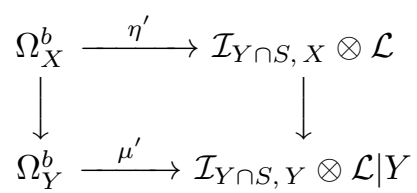

in which $\mu^{\prime}$ is surjective. Indeed, the image of $\eta$ is $\mathcal{I}_{S, X} \otimes \mathcal{L}$ owing to the definition of $S$. So the image of $\Omega_{X}^{b}$ in $\mathcal{L} \mid Y$ is $\mathcal{I}_{Y \cap S, Y} \otimes \mathcal{L} \mid Y$. Since $\Omega_{X}^{b} \rightarrow \Omega_{Y}^{b}$ is surjective, the image of $\mu$ is $\mathcal{I}_{Y \cap S, Y} \otimes \mathcal{L} \mid Y$ too; whence, $\mu$ induces $\mu^{\prime}$. Finally, the natural map $\mathcal{I}_{S, X} \rightarrow \mathcal{I}_{Y \cap S, Y}$ factors through $\mathcal{I}_{Y \cap S, X}$; whence, $\eta$ induces $\eta^{\prime}$.

Since $Y$ is of dimension $b$ and reduced, $\Omega_{Y}^{b}$ is invertible in codimension 0 . Since no $b$-dimensional component of $Y$ lies in $S$, also $\mathcal{I}_{Y \cap S, Y} \otimes \mathcal{L} \mid Y$ is invertible in codimension 0. Hence, since $\mu^{\prime}$ is surjective, $\mu^{\prime}$ is bijective in codimension 0 . So the support of its kernel has dimension at most $b-1$. Therefore, $\mathrm{H}^{b}\left(\mu^{\prime}\right)$ is bijective. Now, $\mathrm{H}^{b}\left(\Omega_{X}^{b}\right) \rightarrow \mathrm{H}^{b}\left(\Omega_{Y}^{b}\right)$ is nonzero. Hence, since Diagram (3.3.1) is commutative,

$$
\mathrm{H}^{b}\left(\eta^{\prime}\right) \neq 0
$$

Thus $\mathrm{H}^{b}\left(\mathcal{I}_{Y \cap S, X} \otimes \mathcal{L}\right) \neq 0$, as asserted.

Assume $\mathrm{H}^{b}(\eta)=0$ now. Form the standard exact sequence

$$
0 \rightarrow \mathcal{I}_{Y \cap S, X} \rightarrow \mathcal{O}_{X} \rightarrow \mathcal{O}_{Y \cap S} \rightarrow 0,
$$

tensor it with $\mathcal{L}$, and extract the following exact sequence of cohomology:

$$
\mathrm{H}^{b-1}(\mathcal{L}) \rightarrow \mathrm{H}^{b-1}(\mathcal{L} \mid(Y \cap S)) \rightarrow \mathrm{H}^{b}\left(\mathcal{I}_{Y \cap S, X} \otimes \mathcal{L}\right) \rightarrow \mathrm{H}^{b}(\mathcal{L}) .
$$

By exactness at $\mathrm{H}^{b}\left(\mathcal{I}_{Y \cap S, X} \otimes \mathcal{L}\right)$, the image of $\mathrm{H}^{b-1}(\mathcal{L} \mid(Y \cap S))$ contains the image of $\mathrm{H}^{b}\left(\eta^{\prime}\right)$ since $\mathrm{H}^{b}(\eta)=0$. But, this image is nonzero owing to (3.3.2). Hence, by exactness at $\mathrm{H}^{b-1}(\mathcal{L} \mid(Y \cap S))$, the first map is not surjective; that is, (1) holds.

In particular, $\mathrm{H}^{b-1}(\mathcal{L} \mid Y \cap S) \neq 0$. Hence $\operatorname{dim}(Y \cap S) \geq b-1$. But no $b$-dimensional component of $Y$ lies in $S$. Therefore, $\operatorname{dim}(Y \cap S)=b-1$; that is, (2) holds.

Since the cokernel of $\mu$ is supported on $Y \cap S$, the map $\mathrm{H}^{b}(\mu)$ is surjective. But it is not bijective since Diagram (3.1.2) is commutative, since $\mathrm{H}^{b}(\eta)=0$ 
and since $\mathrm{H}^{b}\left(\Omega_{X}^{b}\right) \rightarrow \mathrm{H}^{b}\left(\Omega_{Y}^{b}\right)$ is nonzero. Thus (3) holds.

Proposition 3.4. Let $X$ be a smooth projective scheme in characteristic 0 . Let $Y \subseteq X$ be a closed subscheme of pure dimension $b$, and $\mathcal{N}$ its normal sheaf. Assume $\operatorname{Pic}(X)=\mathbf{Z}$, and assume there is a closed curve $C \subseteq X$ contained in the smooth locus $U$ of $Y$ such that $\operatorname{deg} \mathcal{N} \mid C>0$. Let $\eta: \Omega_{X}^{b} \rightarrow \mathcal{L}$ be a Pfaff field, $S$ its singular locus. Assume no b-dimensional component of $Y$ lies in $S$, and assume $Y$ is invariant under $\eta$. Then $\operatorname{dim}(Y \cap S)=b-1$.

Proof. Since $Y$ is invariant, $\eta$ induces a map $\mu: \Omega_{Y}^{b} \rightarrow \mathcal{L} \mid Y$ making (3.1.2) commute. And $\mu$ is surjective off $Z:=Y \cap S$.

Let $\mathcal{I}_{Y, X}$ be the ideal of $Y$ in $X$, and consider the second fundamental sequence,

$$
0 \rightarrow \mathcal{I}_{Y, X} / \mathcal{I}_{Y, X}^{2} \rightarrow \Omega_{X}^{1} \mid Y \rightarrow \Omega_{Y}^{1} \rightarrow 0
$$

It is right exact, and is exact on the smooth locus $U$ of $Y$.

Set $n:=\operatorname{dim} X$ and $a:=n-b$. Then (3.4.1) induces an isomorphism

$$
\Omega_{U}^{b} \leadsto \Omega_{X}^{n}\left|U \otimes \bigwedge^{a} \mathcal{N}\right| U .
$$

Set $V:=U-U \cap Z$. Then $\mu \mid V$ is a surjection between invertible sheaves, whence a bijection. So there is an isomorphism $\rho: \Omega_{X}^{n}\left|V \otimes \bigwedge^{a} \mathcal{N}\right| V \simeq \mathcal{L} \mid V$.

Proceeding by way of contradiction, assume $\operatorname{dim} Z \leq b-2$. Then $U \cap Z$ has codimension at least 2 in $U$. Since $U$ is smooth and $\Omega_{X}^{n}\left|U \otimes \bigwedge^{a} \mathcal{N}\right| U$ and $\mathcal{L} \mid U$ are invertible, $\rho$ extends to an isomorphism $\widetilde{\rho}: \Omega_{X}^{n}\left|U \otimes \bigwedge^{a} \mathcal{N}\right| U \stackrel{\mathcal{L}}{\rightarrow} \mid U$.

By hypothesis, $C \subseteq U$ and $\operatorname{deg} \mathcal{N} \mid C>0$. So $\operatorname{deg} \mathcal{L}\left|C>\operatorname{deg} \Omega_{X}^{n}\right| C$. Since $\operatorname{Pic}(X)=\mathbf{Z}$, the sheaf $\mathcal{L} \otimes\left(\Omega_{X}^{n}\right)^{-1}$ is ample. So, by the Kodaira vanishing theorem, $\mathrm{h}^{b}(\mathcal{L})=0$. Now, since the characteristic is 0 , the natural map $\mathrm{H}^{b}\left(\Omega_{X}^{b}\right) \rightarrow \mathrm{H}^{b}\left(\Omega_{Y}^{b}\right)$ is nonzero by Proposition 2.1. Hence $\operatorname{dim}(Y \cap S)=b-1$ by Proposition 3.3, a contradiction.

Lemma 3.5. Let $X$ be a projective scheme, $Y \subset X$ a reduced closed subscheme of pure dimension b. Assume $Y$ is Gorenstein, and has normalcrossings in codimension 1 . Let $r$ be the number of irreducible components of $Y$, and $\omega_{Y}$ its dualizing sheaf. Let $\eta: \Omega_{X}^{b} \rightarrow \mathcal{L}$ be a Pfaff field, $S$ its singular locus. Assume no irreducible component of $Y$ lies in $S$, and assume $Y$ is invariant under $\eta$. Let $m \geq 0$. Then $\mathrm{h}^{0}\left(\omega_{Y}^{m} \otimes \mathcal{L}^{-m} \mid Y\right) \leq r$.

Proof. Since $Y$ is invariant, $\eta$ induces a map $\mu: \Omega_{Y}^{b} \rightarrow \mathcal{L} \mid Y$, which is surjective away from $Y \cap S$. Now, no irreducible component of $Y$ lies in $S$, and $Y$ is reduced of pure dimension $b$. Hence $\mu$ is generically bijective.

Let $f: \widetilde{Y} \rightarrow Y$ be the normalization map, $\lambda: \Omega_{Y}^{b} \rightarrow f_{*} \Omega_{\widetilde{Y}}^{b}$ the induced map. Since $Y$ is reduced, whence generically smooth, $\lambda$ is generically bijective. Now, $Y$ has normal crossings in codimension 1 ; hence, $\lambda$ is also surjective in codimension 1.

Let $\widetilde{\gamma}: \Omega_{\widetilde{Y}}^{b} \rightarrow \omega_{\widetilde{Y}}$ be the "class" map. It is bijective on the smooth locus of $\tilde{Y}$, so in codimension 1 as $\widetilde{Y}$ is normal. Hence the composition $\beta:=f_{*} \widetilde{\gamma} \circ \lambda$ 
is generically bijective and surjective in codimension 1 . Since the kernel of $\beta$ is torsion, but $\mathcal{L} \mid Y$ is torsion free, it follows that $\mu$ factors through $\beta$ in codimension 1. Now, $Y$ is Gorenstein, so Cohen-Macaulay, and $\mathcal{L} \mid Y$ is invertible. Hence $\mu$ factors through $\beta$, yielding a map $\tau: f_{*} \omega_{\widetilde{Y}} \rightarrow \mathcal{L} \mid Y$. And $\tau$ is generically bijective.

Since $f$ is finite, $f_{*} \omega_{\widetilde{Y}}=\operatorname{Hom}\left(f_{*} \mathcal{O}_{\widetilde{Y}}, \omega_{Y}\right)$. Now, $\omega_{Y}$ is dualizing. Hence $f_{*} \mathcal{O}_{\tilde{Y}}=\operatorname{Hom}\left(f_{*} \omega_{\tilde{Y}}, \omega_{Y}\right)$. Applying $\operatorname{Hom}\left(\bullet, \omega_{Y}\right)$ to $\tau$, we get a generically bijective map $\rho: \operatorname{Hom}\left(\mathcal{L} \mid Y, \omega_{Y}\right) \rightarrow f_{*} \mathcal{O}_{\tilde{Y}}$.

Set $\mathcal{M}:=\operatorname{Hom}\left(\mathcal{L} \mid Y, \omega_{Y}\right)$, and consider the composition

$$
\iota: \mathcal{M}^{m} \stackrel{\rho^{\otimes m}}{\longrightarrow}\left(f_{*} \mathcal{O}_{\tilde{Y}}\right)^{\otimes m} \stackrel{\pi}{\longrightarrow} f_{*} \mathcal{O}_{\tilde{Y}}
$$

where $\pi$ is given by multiplication. Since $f$ is birational, $\pi$ is generically bijective. So, as $\rho$ is generically bijective, $\iota$ is too. Hence, since $\mathcal{M}^{m}$ is invertible and $Y$ is reduced, $\iota$ is globally injective. Thus $\mathrm{h}^{0}\left(\mathcal{M}^{m}\right) \leq \mathrm{h}^{0}\left(f_{*} \mathcal{O}_{\tilde{Y}}\right)$. $\operatorname{Buth} h^{0}\left(f_{*} \mathcal{O}_{\tilde{Y}}\right)=h^{0}\left(\mathcal{O}_{\tilde{Y}}\right)=r$. The assertion follows.

Theorem 3.6. Let $X$ be a smooth projective scheme with $\operatorname{Pic}(X)=\mathbf{Z}$. Let $Y \subset X$ be a reduced closed subscheme, and assume $Y$ is a Cartier divisor with normal crossings in codimension 1 . Let $\sigma \in \mathrm{H}^{0}\left(\Omega_{X}^{1} \otimes \mathcal{N}\right)$ be a Pfaff equation, $S$ its singular locus. Assume no irreducible component of $Y$ lies in $S$, and assume $Y$ is a solution of $\sigma=0$. Then $\operatorname{deg} Y \leq \operatorname{deg} \mathcal{N}$; furthermore, $\operatorname{deg} Y<\operatorname{deg} \mathcal{N}$ if $Y$ is smooth in codimension 1 and the characteristic is 0 .

Proof. Since $X$ is smooth and $Y \subseteq X$ is a Cartier divisor, $Y$ is Gorenstein, and its dualizing sheaf $\omega_{Y}$ is given by the formula $\omega_{Y}=\Omega_{X}^{n}(Y) \mid Y$ where $n:=\operatorname{dim} X$.

Let $\eta: \Omega_{X}^{n-1} \rightarrow \mathcal{L}$ be the Pfaff field corresponding to $\sigma$, so $\mathcal{L}:=\Omega_{X}^{n} \otimes \mathcal{N}$. Then

$$
\omega_{Y} \otimes \mathcal{L}^{-1}\left|Y=\mathcal{N}^{-1}(Y)\right| Y .
$$

By Lemma 3.5, there is an integer $r$ such that $\mathrm{h}^{0}\left(\mathcal{N}^{-m}(m Y) \mid Y\right) \leq r$ for every $m \geq 0$. Hence $\mathcal{N}^{-1}(Y)$ is nonpositive because $\operatorname{dim} Y>0$. So $\operatorname{deg} Y \leq \operatorname{deg} \mathcal{N}$, as asserted.

Furthermore, since $Y$ is invariant, $\eta$ induces a map $\mu: \Omega_{Y}^{n-1} \rightarrow \mathcal{L} \mid Y$. And $\mu$ is surjective off $Y \cap S$. Now, $\Omega_{Y}^{n-1}$ is generically invertible because $Y$ is reduced, and $Y-Y \cap S$ is dense in $Y$; hence, $\mu$ is generically injective.

Assume now that $Y$ is smooth in codimension 1. Then the "class" map $\gamma: \Omega_{Y}^{n-1} \rightarrow \omega_{Y}$ is an isomorphism in codimension 1 . On the other hand, since $Y$ is invariant, $\eta$ induces a map $\mu: \Omega_{Y}^{n-1} \rightarrow \mathcal{L} \mid Y$. Since $Y$ is Gorenstein, so Cohen-Macaulay, and since $\mathcal{L} \mid Y$ is invertible, $\mu$ factors through $\gamma$, yielding a map $\tau: \omega_{Y} \rightarrow \mathcal{L} \mid Y$. As $\mu$ is generically injective, so is $\tau$.

Assume $\operatorname{deg} Y=\operatorname{deg} \mathcal{N}$. Then $\mathcal{N}^{-1}(Y)=\mathcal{O}_{X}$ since $\operatorname{Pic}(X)=\mathbf{Z}$. So $\omega_{Y}=\mathcal{L} \mid Y$ by (3.6.1). Hence $\tau$ corresponds to a generically nonzero, everywhere regular function $f$ on $Y$. Since $Y$ is projective, $f$ is locally constant, so everywhere nonzero. Thus $\tau$ is an isomorphism. Hence $\mu$ is, like $\gamma$, an 
isomorphism in codimension 1. Thus $\operatorname{codim}(Y \cap S, Y) \geq 2$.

However, $Y$ is projective and smooth in codimension 1; so the smooth locus of Y contains a (smooth) projective curve by Bertini's Theorem. Hence, by Proposition 3.4, the characteristic must be nonzero. Thus the second assertion is proved.

\section{Projective spaces}

Definition 4.1. Let $X:=\mathbf{P}^{n_{1}} \times \ldots \times \mathbf{P}^{n_{s}}$ and let $\eta: \Omega_{X}^{b} \rightarrow \mathcal{L}$ be a Pfaff field. Call $\eta$ fibered if the $\mathbf{P}^{n_{i}}$ can be grouped to give a decomposition $X=X_{1} \times X_{2}$ such that

(4.1.1) $\operatorname{dim} X_{1}=b$,

(4.1.2) $\mathcal{L}=\Omega_{X_{1}}^{b} \otimes \mathcal{M}_{2}$ where $\mathcal{M}_{2}$ is nonnegative on $X_{2}$, and

(4.1.3) $\eta: \Omega_{X}^{b} \rightarrow \Omega_{X_{1}}^{b} \otimes \mathcal{O}_{X_{2}} \rightarrow \mathcal{L}$ where the first map is the natural surjection and the second arises from a section of $\mathcal{M}_{2}$.

Lemma 4.2. Let $X:=\mathbf{P}^{n_{1}} \times \ldots \times \mathbf{P}^{n_{s}}$ and let $\eta: \Omega_{X}^{b} \rightarrow \mathcal{L}$ be a Pfaff field. Then $\eta$ is fibered if and only if $\mathrm{H}^{b}(\mathcal{L}) \neq 0$.

Proof. Say $\mathcal{L}=\mathcal{O}_{X}\left(m_{1}, \ldots, m_{s}\right)$. By the Künneth formula,

$$
\mathrm{H}^{b}\left(\mathcal{O}_{X}\left(m_{1}, \ldots, m_{s}\right)\right)=\sum \mathrm{H}^{b_{1}}\left(\mathcal{O}_{\mathbf{P}^{n_{1}}}\left(m_{1}\right)\right) \otimes \cdots \otimes \mathrm{H}^{b_{s}}\left(\mathcal{O}_{\mathbf{P}^{n_{s}}}\left(m_{s}\right)\right)
$$

where the sum ranges over all $s$-tuples $\left(b_{1}, \ldots, b_{s}\right)$ of nonnegative integers $b_{i}$ such that $b_{1}+\cdots+b_{s}=b$. So $\mathrm{H}^{b}\left(\mathcal{O}_{X}\left(m_{1}, \ldots, m_{s}\right)\right) \neq 0$ if and only if there is such an $s$-tuple $\left(b_{1}, \ldots, b_{s}\right)$ such that $\mathrm{H}^{b_{i}}\left(\mathcal{O}_{\mathbf{P}^{n_{i}}}\left(m_{i}\right)\right) \neq 0$ for all $i$. However, $\mathrm{H}^{b_{i}}\left(\mathcal{O}_{\mathbf{P}^{n_{i}}}\left(m_{i}\right)\right) \neq 0$ if and only if either $b_{i}=n_{i}$ and $m_{i} \leq-n_{i}-1$ or $b_{i}=0$ and $m_{i} \geq 0$.

Reorder the $\mathbf{P}^{n_{i}}$ so that $m_{i}<0$ for $1 \leq i \leq t$ and $m_{i} \geq 0$ for $t+1 \leq i \leq s$. Then $\mathrm{H}^{b}(\mathcal{L}) \neq 0$ if and only if

$$
b=n_{1}+\cdots+n_{t} \text { and } m_{i} \leq-n_{i}-1 \text { for } 1 \leq i \leq t .
$$

(Up to this point, $\eta$ has played no role.)

Suppose $\eta$ is fibered. Then, owing to (4.1.2), the $\mathbf{P}^{n_{i}}$ must be grouped into the first $t$ for $X_{1}$ and the rest for $X_{2}$. Also, (4.1.1) and (4.1.2) yield (4.2.1). Hence $\mathrm{H}^{b}(\mathcal{L}) \neq 0$.

Conversely, suppose $\mathrm{H}^{b}(\mathcal{L}) \neq 0$; then (4.2.1) holds. Group the $\mathbf{P}^{n_{i}}$ into the first $t$ for $X_{1}$ and the rest for $X_{2}$ to get $X=X_{1} \times X_{2}$. Then (4.1.1) holds.

According to Definition 3.1, the field $\eta$ corresponds to a nonzero section $\sigma \in \mathrm{H}^{0}\left(\Omega_{X}^{a}\left(m_{1}+n_{1}+1, \ldots, m_{s}+n_{s}+1\right)\right)$ where $a:=n_{1}+\cdots+n_{s}-b$. Now, $\Omega_{X}^{1}$ is equal to the sum of the pullbacks of the sheaves $\Omega_{\mathbf{P}^{n_{i}}}^{1}$. So

$$
\Omega_{X}^{a}=\sum \Omega_{\mathbf{P}^{n_{1}}}^{e_{1}} \otimes \cdots \otimes \Omega_{\mathbf{P}^{n_{s}}}^{e_{s}}
$$

where the sum ranges over all $s$-tuples $\left(e_{1}, \ldots, e_{s}\right)$ of nonnegative integers $e_{i}$ 
such that $e_{1}+\cdots+e_{s}=a$. Via the Künneth formula, $\sigma$ becomes an element of the sum

$$
\sum \mathrm{H}^{0}\left(\Omega_{\mathbf{P}^{n_{1}}}^{e_{1}}\left(m_{1}+n_{1}+1\right)\right) \otimes \cdots \otimes \mathrm{H}^{0}\left(\Omega_{\mathbf{P}_{s}^{n_{s}}}^{e_{s}}\left(m_{s}+n_{s}+1\right)\right) .
$$

Since $\sigma \neq 0$, there is an $s$-tuple $\left(e_{1}, \ldots, e_{s}\right)$ such that

$$
\mathrm{H}^{0}\left(\Omega_{\mathbf{P}^{n_{i}}}^{e_{i}}\left(m_{i}+n_{i}+1\right)\right) \neq 0 \text { for all } i .
$$

This $s$-tuple is unique; in fact, let's now show that

(4.2.3) $e_{i}=0$ and $m_{i}=-n_{i}-1$ for $1 \leq i \leq t$, and

(4.2.4) $e_{i}=n_{i}$ for $t+1 \leq i \leq s$.

Indeed, first fix $i \leq t$. Then $m_{i}+n_{i}+1 \leq 0$ by (4.2.1). Now, $\Omega_{\mathbf{P}^{n_{i}}}^{e_{i}}$ embeds in a direct sum of copies of $\mathcal{O}_{\mathbf{P}^{n_{i}}}\left(-e_{i}\right)$; hence, $\mathrm{H}^{0}\left(\Omega_{\mathbf{P}^{n_{i}}}^{e_{i}}\left(m_{i}+n_{i}+1\right)\right)$ is nonzero only if $m_{i}+n_{i}+1-e_{i} \geq 0$. But, $e_{i} \geq 0$. Therefore, (4.2.3) holds.

Owing to (4.2.3), we have $e_{t+1}+\cdots+e_{s}=a$. However, $a=n_{t+1}+\cdots+n_{s}$ again owing to (4.2.1). Since $e_{i} \leq n_{i}$ for all $i$, therefore (4.2.4) holds.

By (4.2.1) and (4.2.3), $\mathcal{L}=\Omega_{X_{1}}^{b} \otimes \mathcal{M}_{2}$, where $\mathcal{M}_{2}:=\mathcal{O}_{X_{2}}\left(m_{t+1}, \ldots, m_{s}\right)$. Since $m_{i} \geq 0$ for $t+1 \leq i \leq s$, the sheaf $\mathcal{M}_{2}$ is nonnegative. So (4.1.2) holds

Owing to (4.2.3) and (4.2.4), the sum in (4.2.2) reduces to the single term

$$
\begin{aligned}
& \mathrm{H}^{0}\left(\mathcal{O}_{\mathbf{P}^{n_{1}}}\right) \otimes \cdots \otimes \mathrm{H}^{0}\left(\mathcal{O}_{\mathbf{P}^{n_{t}}}\right) \\
& \quad \otimes \mathrm{H}^{0}\left(\Omega_{\mathbf{P}^{n_{t+1}}}^{n_{t+1}}\left(m_{t+1}+n_{t+1}+1\right)\right) \otimes \cdots \otimes \mathrm{H}^{0}\left(\Omega_{\mathbf{P}^{n_{s}}}^{n_{s}}\left(m_{s}+n_{s}+1\right)\right) .
\end{aligned}
$$

This term is, by the Künneth formula, equal to

$$
\mathrm{H}^{0}\left(\mathcal{O}_{X_{1}} \otimes \Omega_{X_{2}}^{a}\left(m_{t+1}+n_{t+1}+1, \ldots, m_{s}+n_{s}+1\right)\right) .
$$

Since $a=n_{t+1}+\cdots+n_{s}$, the latter corresponds to $\mathrm{H}^{0}\left(\mathcal{M}_{2}\right)$. Thus $\sigma$ corresponds to a section of $\mathcal{M}_{2}$, and (4.1.3) follows.

Theorem 4.3. Set $X:=\mathbf{P}^{n_{1}} \times \ldots \times \mathbf{P}^{n_{s}}$. Let $Y \subseteq X$ be a reduced closed subscheme of dimension $b$ and of multidegree $\left(d_{1}, \ldots, d_{s}\right)$. Assume either the characteristic is 0 , or it is $p>0$ and $p$ does not divide some $d_{i}$. Let $\eta: \Omega_{X}^{b} \rightarrow \mathcal{L}$ be a Pfaff field, and $S$ its singular locus. Assume no $b$-dimensional component of $Y$ lies in $S$, and $Y$ is invariant under $\eta$.

(1) Then $\mathrm{H}^{b}\left(\mathcal{I}_{Y \cap S, X} \otimes \mathcal{L}\right) \neq 0$ where $\mathcal{I}_{Y \cap S, X}$ is the ideal of $Y \cap S$ in $X$.

(2) If $\eta$ is nonfibered, then $\operatorname{dim}(Y \cap S)=b-1$.

(3) If $\eta$ is nonfibered and $\mathrm{h}^{b}\left(\Omega_{Y}^{b}\right)=1$, then $\mathrm{h}^{b}(\mathcal{L} \mid Y)=0$.

Proof. The natural map $\mathrm{H}^{b}\left(\Omega_{X}^{b}\right) \rightarrow \mathrm{H}^{b}\left(\Omega_{Y}^{b}\right)$ is nonzero either by Proposition 2.1 or by Proposition 2.2. Hence Proposition 3.3 yields (1).

Assume $\eta$ is nonfibered. Then $\mathrm{H}^{b}(\mathcal{L})=0$ by Lemma 4.2 , so $\mathrm{H}^{b}(\eta)=0$. Hence Proposition 3.3 yields (2). It also yields that $\mathrm{h}^{b}\left(\Omega_{Y}^{b}\right)>\mathrm{h}^{b}(\mathcal{L} \mid Y)$; whence, (3) holds. 
Remark 4.4. Let $X:=\mathbf{P}^{n_{1}} \times \ldots \times \mathbf{P}^{n_{s}}$, and $Y \subseteq X$ be a reduced connected closed subscheme of pure dimension $b$. Let $\eta: \bar{\Omega}_{X}^{b} \rightarrow \mathcal{L}$ be a fibered Pfaff field, and $S$ its singular locus. Note that (4.1.3) implies $S=X_{1} \times S_{2}$ where $S_{2}$ is a divisor of $X_{2}$. Assume no irreducible component of $Y$ lies in $S$, and $Y$ is invariant under $\eta$.

Suppose the characteristic is 0 . Let $Z$ be the image of $Y$ on $X_{2}$ under the projection map, and $\pi: Y \rightarrow Z$ be the induced surjection. Since $Y$ is invariant under $\eta$, by (4.1.3) the differential $d \pi_{y}$ is zero at $y \in Y-Y \cap S$. Now, since the characteristic is 0 , the induced map $\pi: Y \rightarrow Z$ is generically smooth. As $Y-Y \cap S$ is dense in $Y$, the map $d \pi_{y}$ is zero for a general $y \in Y$. Hence $\operatorname{dim} Z=0$, and since $Y$ is connected, $Z=\{P\}$ for $P \in X_{2}-S_{2}$. So $Y=X_{1} \times\{P\}$. Hence $Y \cap S$ is empty; so (2) of Theorem 4.3 fails. Furthermore, $\mathcal{L} \mid Y=\Omega_{Y}^{b}$; so (3) fails as well.

Corollary 4.5. Let $X:=\mathbf{P}^{n}$, and $Y \subseteq X$ a reduced closed subscheme of dimension $b$ and of degree $d$. Assume either the characteristic is 0 or it is $p>0$ and $p$ does not divide d. Let $\eta: \Omega_{X}^{b} \rightarrow \mathcal{L}$ be a Pfaff field, and $S$ its singular locus. Assume no b-dimensional component of $Y$ lies in $S$, and $Y$ is invariant under $\eta$. Set $l:=\operatorname{deg} \mathcal{L}$. Then

$$
\operatorname{reg}(Y \cap S)>b+l \text { and } \operatorname{dim}(Y \cap S)=b-1
$$

where $\operatorname{reg}(Y \cap S)$ denotes the Castelnuovo-Mumford regularity.

Assume $\mathrm{h}^{b}\left(\Omega_{Y}^{b}\right)=1$ in addition. Then $\mathrm{h}^{b}(\mathcal{L} \mid Y)=0$. Furthermore, if $Y$ is arithmetically Cohen-Macaulay, then $\operatorname{reg} Y \leq b+l+1$; if $Y$ is s-subcanonical, then $s \leq l-1$.

Proof. Note that $\eta$ is nonfibered by Definition 4.1 since $0<b<n$. Hence the first three assertions are immediate consequences of those of Theorem 4.3.

Set $m:=b+l+1$. Then $m \geq 0$. Indeed, $\eta$ corresponds to a nonzero section of $\Omega_{X}^{n-b}(l+n+1)$. Since $\Omega_{X}^{n-b}$ embeds in a direct sum of copies of $\mathcal{O}_{X}(b-n)$, we get a nonzero section of $\mathcal{O}_{X}(m)$. So $m \geq 0$.

By definition, $\operatorname{reg} Y \leq m$ if $\mathrm{H}^{i}\left(\mathcal{I}_{Y, X}(m-i)\right)$ vanishes for $i \geq 1$.

Consider the standard exact sequence

$\mathrm{H}^{i-1}\left(\mathcal{O}_{X}(m-i)\right) \rightarrow \mathrm{H}^{i-1}\left(\mathcal{O}_{Y}(m-i)\right) \rightarrow \mathrm{H}^{i}\left(\mathcal{I}_{Y, X}(m-i)\right) \rightarrow \mathrm{H}^{i}\left(\mathcal{O}_{X}(m-i)\right)$.

Since $m \geq 0$, the last group vanishes for $i \geq 1$. For $i=b+1$, the second group is $\mathrm{H}^{b}(\mathcal{L} \mid Y)$, hence zero by the third assertion. So $\mathrm{H}^{i}\left(\mathcal{I}_{Y, X}(m-i)\right)$ vanishes for $i=b+1$.

Suppose $Y$ is arithmetically Cohen-Macaulay. Then the first map in (4.5.1) is surjective for $i=1$, and $\mathrm{H}^{i-1}\left(\mathcal{O}_{Y}(m-i)\right)$ vanishes for $i \neq 1, b+1$. So $\mathrm{H}^{i}\left(\mathcal{I}_{Y, X}(m-i)\right)$ vanishes for $i \neq 0, b+1$ as well. Thus reg $Y \leq m$.

Finally, assume $Y$ is $s$-subcanonical; that is, $\mathcal{O}_{Y}(s)$ is its dualizing sheaf. Now, $\mathrm{h}^{b}(\mathcal{L} \mid Y)=0$, and $\mathcal{L} \mid Y=\mathcal{O}_{Y}(l)$. Hence $\mathrm{h}^{0}\left(\mathcal{O}_{Y}(s-l)\right)=0$ by duality. So $s-l \leq-1$. 
Remark 4.6. Let $X:=\mathbf{P}^{n}$, and $Y \subseteq X$ a reduced closed subscheme of dimension $b$. Assume $\mathrm{h}^{b}\left(\Omega_{Y}^{b}\right)=1$. Let $\omega_{Y}$ be the dualizing sheaf. Then, by duality,

$$
\mathrm{H}^{b}\left(\Omega_{Y}^{b}\right)=\operatorname{Hom}\left(\Omega_{Y}^{b}, \omega_{Y}\right)^{*} \text { and } \mathrm{H}^{b}\left(\Omega_{Y}^{b}(1)\right)=\operatorname{Hom}\left(\Omega_{Y}^{b}, \omega_{Y}(-1)\right)^{*} .
$$

Take any nonzero map $\gamma: \Omega_{Y}^{b} \rightarrow \omega_{Y}$, and let $T$ be the support of its cokernel. Take a hyperplane $H$ that doesn't contain $T$. Then the image of $\gamma$ is not contained in $\omega_{Y}(-H)$. So $\gamma \notin \operatorname{Hom}\left(\Omega_{Y}^{b}, \omega_{Y}(-H)\right)$. Hence $\operatorname{Hom}\left(\Omega_{Y}^{b}, \omega_{Y}(-1)\right)=0$. Thus $\mathrm{h}^{b}\left(\Omega_{Y}^{b}(1)\right)=0$.

Let $\eta: \Omega_{X}^{b} \rightarrow \mathcal{L}$ be a Pfaff field, and $S$ its singular locus. Assume $Y$ is invariant under $\eta$, and form the map $\mu: \Omega_{Y}^{b} \rightarrow \mathcal{L} \mid Y$ induced by $\eta$; see Diagram (3.1.2). Assume no $b$-dimensional component of $Y$ lies in $S$. Then $\mu$ is surjective off $Y \cap S$; furthermore, $\operatorname{dim} Y \cap S \leq b-1$. Since $\mathrm{h}^{b}\left(\Omega_{Y}^{b}(1)\right)=0$, it follows that $\mathrm{h}^{b}(\mathcal{L}(1) \mid Y)=0$.

Set $l:=\operatorname{deg} \mathcal{L}$. The proof of Corollary 4.5 now yields these two weaker conclusions: if $Y$ is arithmetically Cohen-Macaulay, then $\operatorname{reg} Y \leq b+l+2$; if $Y$ is $s$-subcanonical, then $s \leq l$.

For the stronger conclusions of Corollary 4.5 to hold however, it is necessary that either the characteristic be 0 or it be $p>0$ and $p$ not divide $d$ where $d:=\operatorname{deg} Y$. For example, take $n:=2$ and $p>0$, and let $Y$ be a smooth curve such that $p$ divides $d$. Then it is possible to find a Pfaff field $\eta: \Omega_{X}^{1} \rightarrow \mathcal{L}$ where $\mathcal{L}:=\mathcal{O}_{X}(d-3)$ such that $Y$ is invariant under $\eta$; see Remark 14 on p. 8 of [E].

Remark 4.7. Let $Y$ be an integral projective scheme of dimension $b$. Suppose that $Y$ has normal-crossings singularities in codimension 1; that is, there is a closed set $F$ of dimension at most $b-2$ such that $Y-F$ has two sheets meeting transversally. Let's show $\mathrm{h}^{b}\left(\Omega_{Y}^{b}\right)=1$.

Indeed, let $f: Y^{*} \rightarrow Y$ be the normalization map. The natural map $\Omega_{Y}^{1} \rightarrow f_{*} \Omega_{Y^{*}}^{1}$ is bijective off $F$. Since $\operatorname{dim} F \leq b-2$, the induced map $\mathrm{H}^{b}\left(\Omega_{Y}^{b}\right) \rightarrow \mathrm{H}^{b}\left(f_{*} \Omega_{Y^{*}}^{b}\right)$ is bijective. Its target is equal to $\mathrm{H}^{b}\left(\Omega_{Y^{*}}^{b}\right)$ as $f$ is finite. Thus we may assume $Y$ is normal.

Consider the "class" map from $\Omega_{Y}^{b}$ to the dualizing sheaf $\omega_{Y}$. This map is bijective on the smooth locus of $Y$. Since the singular locus of $Y$ has dimension at most $b-2$, because $Y$ is normal, the class map induces a bijection from $\mathrm{H}^{b}\left(\Omega_{Y}^{b}\right)$ to $\mathrm{H}^{b}\left(\omega_{Y}\right)$. The latter group is, by duality, equal to $H^{0}\left(\mathcal{O}_{Y}\right)$. Since $Y$ is integral, $h^{0}\left(\mathcal{O}_{Y}\right)=1$. Thus $\mathrm{h}^{b}\left(\Omega_{Y}^{b}\right)=1$.

\section{ACKNOWLEDGMENTS}

The first author thanks A. Campillo, L. G. Mendes, P. Sad, M. Soares, and especially J. V. Pereira for helpful discussions on the subject. He is also grateful to CNPq for a grant, Proc. 202151/90-5, supporting a yearlong visit to MIT, and grateful to MIT for its hospitality. He was also 
supported by PRONEX, Convênio 41/96/0883/00, CNPq, Proc. 300004/958, and FAPERJ, Proc. E-26/170.418/2000-APQ1.

The second author thanks IMPA, Rio de Janeiro, ICMC-USP, São Carlos, and the XVI and XVII Escolas de Álgebra, Brasília and Cabo Frio, for their invitations and financial support, which enabled this work to be initiated, pursued, and presented.

\section{REFERENCES}

[BM] Brunella, M. and Mendes, L. G., Bounding the degree of solutions to Pfaff equations, Publ. Mat. 44 (2000), 593-604.

[CCG] Campillo, A., Carnicer, M. and García de la Fuente, J., Invariant curves by vector fields on algebraic varieties, J. London Math. Soc. 62 (2000), 56-70.

[CL] Cerveau, D. and Lins Neto, A., Holomorphic foliations in $\mathbf{P}_{\mathbf{C}}^{2}$ having an invariant algebraic curve, Ann. Inst. Fourier 41 (1991), 883-903.

[dPW] du Plessis, A. A. and Wall, C. T. C. Application of the theory of the discriminant to highly singular plane curves, Mat. Proc. Camb. Phil. Soc. 126 (1999), 259-266.

[E] Esteves, E., The Castelnuovo-Mumford regularity of an integral variety of a vector field on projective space, Math. Res. Lett. 9 (2002), 1-15.

[EK1] Esteves E. and Kleiman, S., Bounds on leaves of one-dimensional foliations, Preprint 2002.

[EK2] Esteves, E. and Kleiman, S., Bounds on leaves of foliations of the plane, Preprint 2003.

[J] Jouanolou, J.P., Equations de Pfaff algébriques, Lecture Notes in Mathematics 708, Springer, 1979.

[K] Kleiman, S., The transversality of a general translate, Comp. Math. 28 (1974), $287-297$.

[Le] Lehmann, D., Résidus des sous-varietés invariantes d'un feuilletage singulier, Ann. Inst. Fourier 41 (1991), 211-258.

[Li] Lipman, J., Dualizing sheaves, differentials and residues on algebraic varieties, Astérisque 117 (1984).

[M] M. McQuillan, Diophantine approximations and foliations, Inst. Hautes Études Sci. Publ. Math. 87 (1998), 121-174.

[P] Poincaré, H., Sur l'intégration algébrique des équations differentielles du premier ordre et du premier degré, Rendiconti del Circolo Matematico di Palermo 5 (1891), 161-191.

[S] Soares, M., The Poincaré problem for hypersurfaces invariant by one-dimensional foliations, Invent. Math. 128 (1997), 495-500. 